\title{
Effect of the Silicon Drift Detector on EDAX Standardless Quant Methods
}

\section{Frank Eggert}

EDAX Inc. Materials Analysis Division, AMETEK, 91 McKee Drive, Mahwah, NJ 07430

frank.eggert@ametek.com

\begin{abstract}
Two standardless quantitative methods for evaluating EDS $\mathrm{X}$-ray spectra were investigated in regards their basic metrics. Both methods have similar total errors, but the error contributions are from different sources. In the P/B-based method, error is more related to counting statistics and therefore can benefit from high count rates achievable with modern silicon drift detectors. To reduce systematic uncertainties in the net-count-based standardless approach, measured values need to be supported by data in a previously measured database. Using the P/B-based method, it is now possible to achieve standardless EDS quantification within $\pm 10 \%$ relative deviation from true composition for $95 \%$ of results.
\end{abstract}

Keywords: X-ray spectra, standardless EDS quantification, silicon drift detector, P/B PeBaZAF Quant, eZAF Quant

\section{Introduction}

When used with a scanning electron microscope (SEM), energy-dispersive X-ray spectrometry (EDS) offers the microscopist significant analytical capabilities. For example, the operator can obtain distribution maps that are color-coded according to elements or phases present in the analyzed material. If more analytical details are needed, it is necessary to analyze the X-ray spectra from each distinguishable multi-element phase. From these spectra, quantitative values for the amounts of elements within each phase can be estimated. Sophisticated physical models, algorithms, and computer programs are needed to do this. The resultant uncertainties are a combination of the "precision" of the measured data and the "accuracy" available from the measurement setup, fundamental atomic databases, and the correctness of the matrix-correction models employed to convert X-ray peak intensities into values for the amounts of elements in the specimen. While standardless quantitative analysis had been developed and accomplished for many years using conventional $\mathrm{Si}(\mathrm{Li})$ EDS detection systems, the advent of silicon drift detectors (SDDs) has the potential to improve this type of analysis. Since the SDD can count over 100 times faster than the older $\mathrm{Si}(\mathrm{Li})$ detector, the SDD can quickly provide enough counts in X-ray peaks and background to significantly reduce the experimental scatter and improve the "precision" of the measurements.

In this article two standardless methods used in EDAX software are compared without and with the high-count rate provided by an SDD. One of these methods, which depends more on the number of counts collected (counting statistics), benefits significantly from the SDD.

\section{Materials and Methods}

Element identification is the first step toward getting a proper view of the sample composition. No Quant model would be able to calculate the element concentrations properly if the assumption about which elements are present in the sample is incorrect or wrongly evaluated. This is why EXpertID [1] is used with all EDAX spectra evaluation software to automatically identify the elements present. This procedure includes iterative evaluation of the residual spectrum, which is the measured minus the reconstructed spectrum, to find small peaks which were not detected in other ways.

Data from the spectrum. Figure 1 shows a simulated [2] spectrum of $\mathrm{GaP}$ with indications of how raw data are obtained for the two different Quant methods used in EDAX standardless analysis software (following the definitions in [3]). One method is based on evaluation of the measured net-counts of element peaks above the background $(\mathrm{P}=$ measured counts minus the background, shown in "green"). The other method is based on peak-to-background ratios $(\mathrm{P} / \mathrm{B}=\mathrm{P}$ divided by background, shown as "green" divided by "purple"). In both cases, the measured $\mathrm{P}$ and $\mathrm{P} / \mathrm{B}$ values are greater with higher element concentrations in the sample. But there is usually no linear relationship between element concentration and measured element $\mathrm{X}$-ray signal; moreover, other elements can cause large inter-element effects. For both methods, the measured input values for the quantification calculation (net intensities or $\mathrm{P} / \mathrm{B}$ ) require the $\mathrm{X}$-ray background to be subtracted from the gross peak. In the case of the P/B method, the peak intensity must also be divided by the background; thus, accurate background values are particularly important. If element lines overlap, a deconvolution of the peaks is required.

Among the methods for determining the background, EDAX uses the physics-based bremsstrahlung calculation $[4,5]$ and a pure mathematical background approximation method,

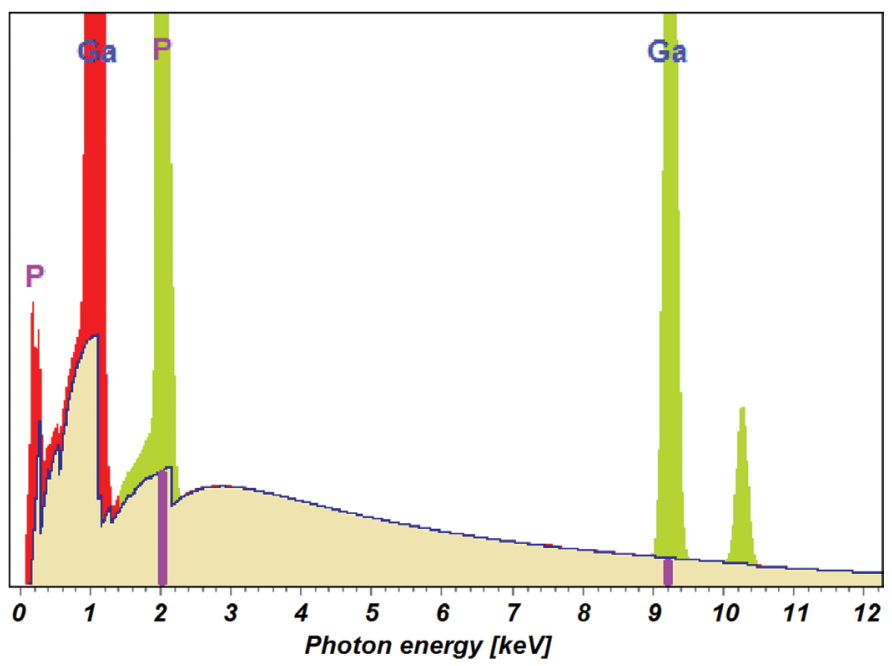

Figure 1: Two different quantification models, one based on evaluation of the measured net-counts of element peaks (green) and the other based on peak-tobackground $(\mathrm{P} / \mathrm{B})$ ratios (green divided by purple). 


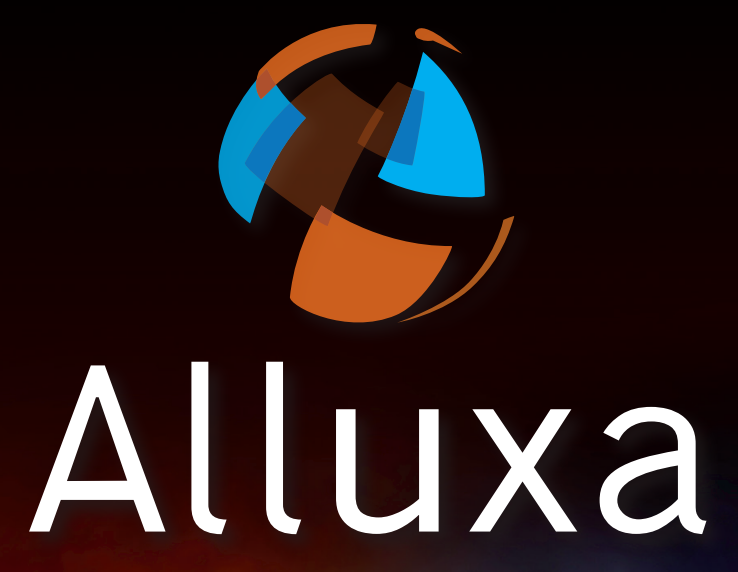

OPTICAL COATINGS REDEFINED

\section{YOUR OPTICAL COATING PARTNER}

alluxa.com

\section{8 lumencor}

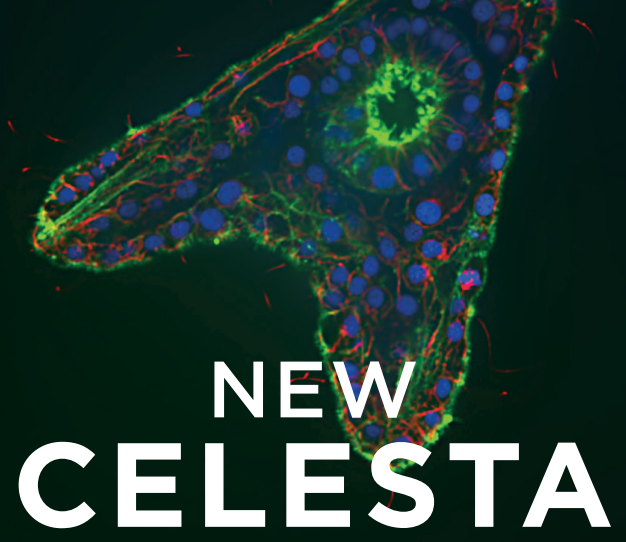

7-LINE LASER LIGHT ENGINE

THE NEXT GENERATION COMES TO LIGHT

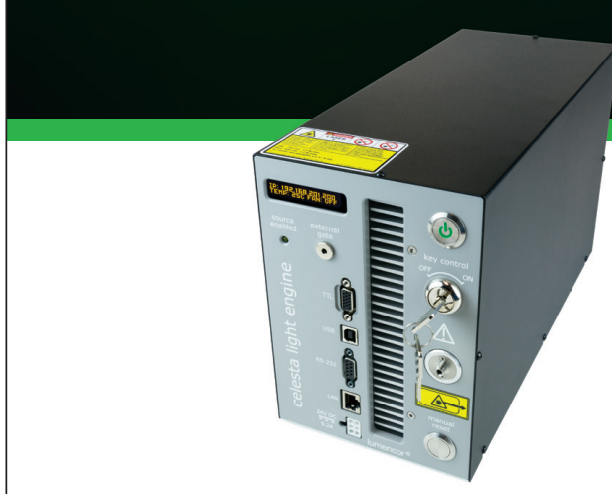

- Spectral breadth: 405, 446, 477, 520, 546, 638, 749nm

- Power: $1000 \mathrm{~mW} / \mathrm{color}$ at distal end of $1.5 \mathrm{~mm}$ fiber, $\sim 7$ Wtotal

- Control: Active power stabilization

- Stability: Exceptional reproducibility, ideal for quantitation

- Ease of use: Pre-aligned, independent lasers

- Applications: Confocal, MERFISH, super-resolution microscopy, photoactivation, optogenetics, FRAP, DNA-PAINT and more

- Off-the-shelf and custom configurations upon request

\section{www.lumencor.com}


SNIP [6]. Mathematical background methods are not suitable for P/B based quantification [4] because the absorption jumps at large peaks are not easily determined, but knowledge of them is required for correct calculation of proper $\mathrm{P} / \mathrm{B}$ values. Peak deconvolution is performed with a probability-theorybased Bayesian algorithm $[7,8]$. Background calculation and peak deconvolution procedures are also core components of the EXpertID initial qualitative analysis of measured spectra. Also required are incomplete charge collection and pile-up corrections to the spectrum; these are related to the detector properties and the pulse processing electronics.

Corrections for matrix effects. Two methods are available in EDAX software to convert the raw data ( $\mathrm{P}$ or $\mathrm{P} / \mathrm{B})$ to element concentrations for bulk samples: the net-counts procedure called eZAF and the P/B-based termed PeBaZAF. Both correction procedures employ aspects of the well-known ZAF corrections [9]. The ZAF abbreviation stands for atomic number correction (Z), absorption correction (A), and secondary fluorescence correction (F).

ZAF method basics. A classical (very simplified) formula, where $N_{\mathrm{i}}$ stands for the measured net-counts of characteristic radiation (ch) and $c_{i}$ are the unknown weight fractions for elements $i$, is:

$$
N_{a, i}^{c h}=c_{a, i} \varepsilon_{i} \text { it } \frac{d \Omega}{4 \pi} \omega_{i} q_{i} \quad(Z A F)_{a, i}^{c h}
$$

where $\varepsilon_{i}$ is the detector efficiency, and it $\frac{d \Omega}{4 \pi}$ is the product of the electron microscope beam-current, the measurement time, and the solid angle. The index $a$ stands for "all" elements and indicates those values that depend on the amounts of the other elements in the specimen. $\omega_{i}$ is the fluorescence yield, the fraction of ionizations that result in emission of an X-ray photon, and $q_{i}$ is the transition probability of an evaluated line in a line series (for example, the K $\alpha$ part of $\mathrm{K}$-series). An iteration is required to solve the equation system. In Equation 1, the $\mathrm{Z}$ describes the generated X-rays by primary electron interactions inside of the sample. The $\mathrm{A}$ and $\mathrm{F}$ factors consider the loss of $\mathrm{X}$-rays due to absorption in the sample and the enhancement by secondary fluorescence caused by other generated X-rays with higher photon energies. But with classical standards-based notation, the measured net counts of the unknown sample are always related to the measured net counts from a standard sample; this is the k-ratio of standards-based analysis. We can modify Equation 1 to:

$$
\frac{N_{a, i}^{s}}{N_{i}^{s t}}=k_{a, i}=c_{a, i} \frac{(Z A F)_{a, i}^{s}}{(Z A F)_{i}^{s t}}
$$

Where $s$ is for the sample and $s t$ stands for standard, usually a pure element standard, measured under the same conditions. Several factors cancel out because they are usually the same for measurements of the standard and the sample. With further simplification Equation 2 becomes:

$$
k_{a, i}=c_{a, i}\left(Z A F^{\prime}\right)_{a, i}
$$

The $Z A F^{\prime}$ factors are now all correction factors relative to the pure element standard. It is possible to take the k-ratio and divide by $Z A F^{\prime}$ factors to get the weight fractions of the elements in the unknown. The $Z A F^{\prime}$ notation of Equation 3 is still used in standardless quantification results to keep it somehow consistent with the classical standards-based formula. Nevertheless, since no standards were measured, the pure-element-related k-ratio is actually calculated via a model; it is not a really a measured value.

The eZAF method. In this article a generically standardless-based correction factor notation is always used. The atomic number correction $\mathrm{Z}$, which is in two parts, is no longer a "correction" but a calculation of primarily excited X-rays [9]. There is a calculated integral over energy-dependent cross sections that considers the stopping power for electrons in the sample, the energy lost from incident electrons. This is the S-factor calculation for all electron-generated X-ray photons. The second part of the $\mathrm{Z}$ factor is a separate backscatter "correction," the R-factor $[3,9]$, which compensates for electrons escaping the sample and not generating X-rays. One can derive from Equation (1):

$$
N_{a, i}^{c h}=c_{a, i} \varepsilon_{i} i t \frac{d \Omega}{4 \pi} \omega_{i} q_{i} S_{a, i}^{c h}(R A F)_{a, i}^{c h}
$$

The dead-time corrected measurement time is known, but it is not usually possible to determine the beam-current exactly in an SEM. There is one more unknown parameter than the available equations, thus it is necessary to use the additional constraint that the sum of all concentrations must equal $100 \%$. But it is possible to determine the unknown parameter indirectly with a separate reference measurement on a pure element sample under the same conditions. In the latter case, the results can be calculated as un-normalized compositions.

The eZAF method uses a $\mathrm{Z}$ and $\mathrm{R}$ calculation that follows the fundamental work by Love \& Scott [10]. The absorption correction used is based on the Sewell/Love/Scott "Quadrilateral" model for estimating the curve of generated X-rays as a function of depth [11]. In the absorption correction, a more recent database of mass absorption coefficients (MACs) by Elam et al. [12] is also used. This is important because these MAC values can strongly affect the absorption correction. The fluorescence correction is based on [13].

Figure 2 shows a HfNi sample spectrum, and Figure 3 shows the evaluation of the spectrum with a k-ratio-based result presentation versus generic standardless notation. The internal correction calculation is same using identical models. Therefore, the results are same. The difference in the upper table is that the k-ratio notation is assuming smaller corrections in relation to an estimated pure element measurement. But this measurement was actually not performed and therefore the algorithm has calculated the k-ratio, based on a theoretical model. In the lower table of Figure 3, the correction factors in relation to generated $\mathrm{X}$-rays are actually greater because standard measurements were not performed. Based on different estimated corrections, the calculated systematic error estimations are different. In the standardless notation, all R- and A-corrections are less than 1.0 (a loss from primary excited X-rays), and all F-corrections are greater than 1.0 (an enhancement of primarily excited X-rays). This is not what happens with $\mathrm{k}$-ratio of $Z A F^{\prime}$ notation where everything is compared to pure element standards. In the standardless case, the k-ratio is a fake. It was never really measured.

The P/B method. The EDAX PeBaZAF is a P/B-method following the basic ZAF approaches, but in this case a precise measurement must be made of the bremsstrahlung background. The basic relation is given by writing Equation 4 for 


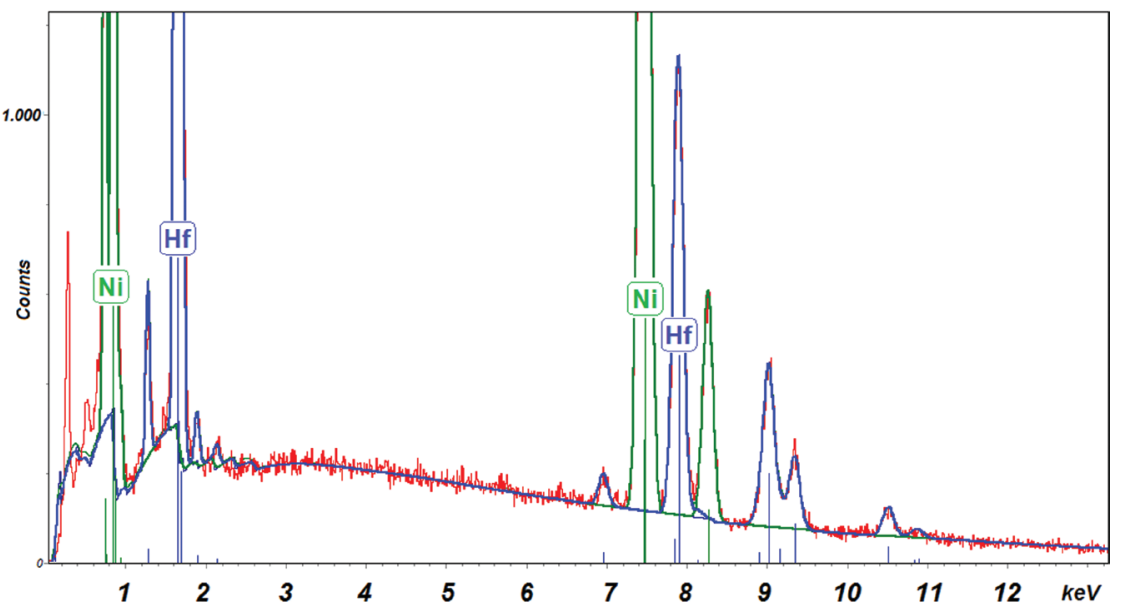

Figure 2: Spectrum from a Ni/Hf sample acquired with an SDD. Accelerating voltage=20 kV; counting time $=30$ seconds; count rate $13 \mathrm{kcps}$. Calculated background and spectrum reconstruction are shown. energy as the peak, and the EDAX software provides this with an iterative bremsstrahlung calculation [4]. But the small number of counts in the measured background intensity (B) significantly influences the value of $\mathrm{P} / \mathrm{B}$, causing the PeBaZAF to exhibit a worse value of precision compared to eZAF. This means that PeBaZAF has poorer repeatability, which is detrimental for comparison tasks. On the other hand, the ZAF correction in the PeBaZAF is smaller (correction factors are closer to 1), leading to fewer systematic errors and good mean accuracy. The eZAF method exhibits larger correction factors, which reduces accuracy, but it has better precision. So one could say these two methods are "complementary" [16].

To evaluate the expected uncertainty with these quantitative measurements, an error parameter was calculated for each result. The parameter Err\% is based on company software both $\mathrm{P}$ and $\mathrm{B}$ and dividing the equations. Thus, $(P / B)_{i}$ represents the number of net characteristic $(c h)$ peak counts divided by the measured bremsstrahlung $(b r)$ at the same energy:

$$
(P / B)_{a, i}=c_{a, i} \omega_{i} q_{i} \frac{S_{a, i}^{c h}}{S_{a, i}^{b r}} \frac{(R A F)_{a, i}^{c h}}{(R A)_{a, i}^{b r}}
$$

Compared to the eZAF formula, some parameters dropped out of Equation 5 so that the number of unknown variables is equal to the number of equations. The solution does not require normalization of the composition values so that they sum to $100 \%$. There is no physical effect to generate bremsstrahlung radiation by other X-rays (all bremsstrahlung is generated by charged particles), thus there is no F-factor for the bremsstrahlung model. This P/B model has been developed and improved from the 1980s until today $[3,13,2,14]$. All ZAF factors within Equation 5, except the F-correction, are ratios between characteristic radiation and bremsstrahlung.

Comparison of the two methods. Both approaches have advantages and disadvantages. The original design idea was that the $\mathrm{P} / \mathrm{B}$ would always be the method of choice if the sample surface is not flat (particles, rough surfaces) because many effects in excitation and absorption cancel out. But during early work with this method, it was found that the P/B produced good accuracy [15] in general, even for flat specimens. The challenge with PeBaZAF method is to extract the proper $\mathrm{P} / \mathrm{B}$ values from a measured spectrum. An accurate background determination is required at the same

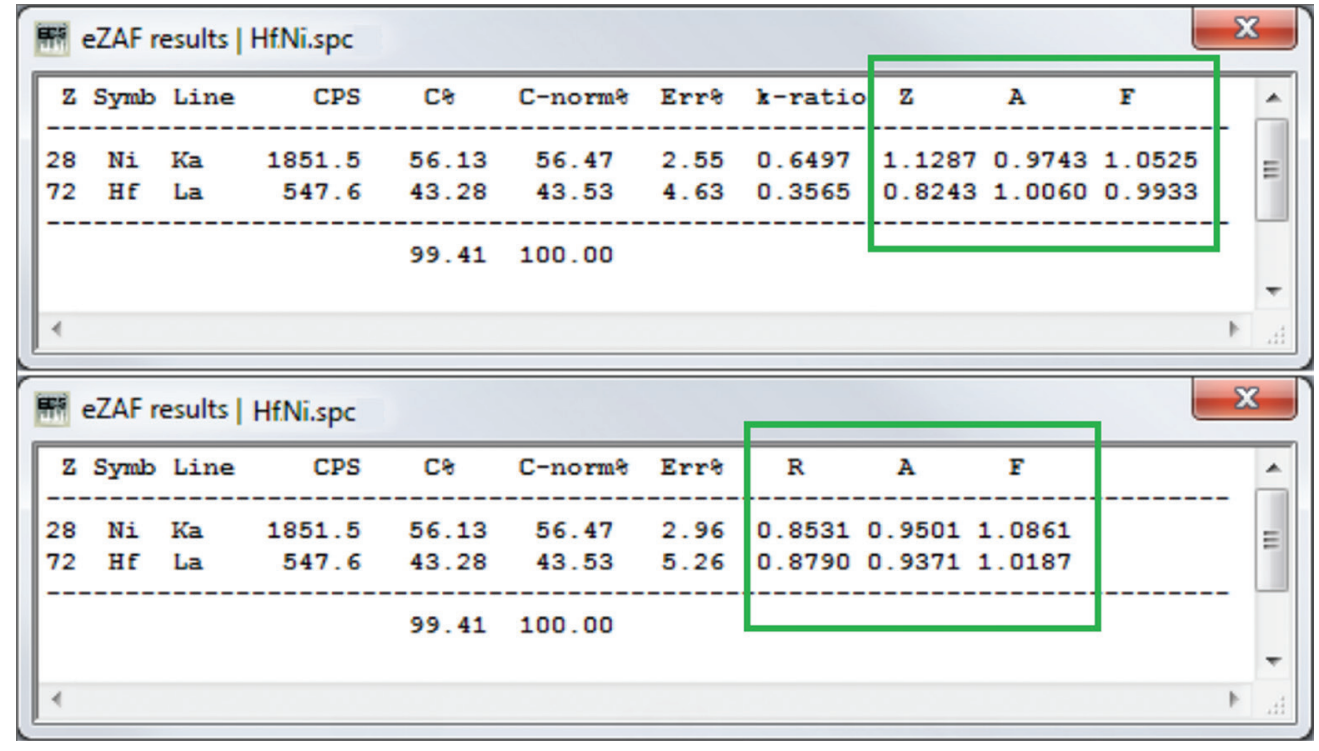

Figure 3: Two ways of representing standardless ZAF results. (top) Net-count-based eZAF analysis of a Ni/Hf sample with "classical" k-ratio notation; all corrections are in relation to estimated "pure" element standards (Equation 3). (bottom) Generic standardless notation where all corrections are in relation to primary electron excited X-rays (Equation 4). The standardless unnormalized results were obtained using a single reference measurement made on pure $\mathrm{Cu}$ to determine the absolute relation. Note that the measurement input and the final analytical results are identical. The difference is only the notation of presented factors. The calculated error is based on different assumptions for each correction. Only the second view is a correct depiction of the corrections employed in standardless case. 

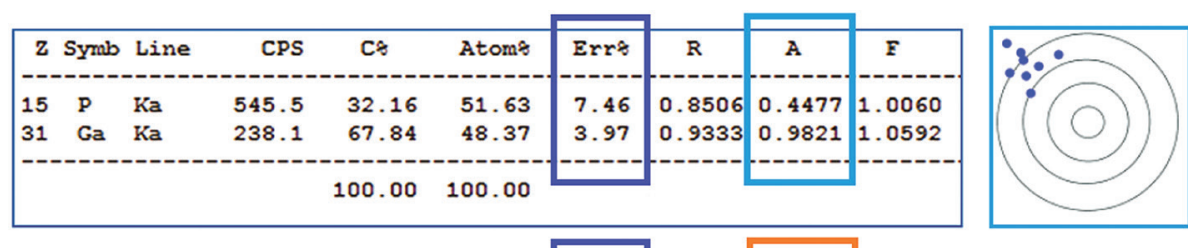

(a)

eZAF

2 kcps

\begin{tabular}{|c|c|c|c|c|c|c|c|c|c|}
\hline & Symb & Line & $\mathrm{P} / \mathrm{B}$ & $\mathrm{Cs}$ & Atoms & Errs & $\mathbf{R}$ & A & $\mathbf{F}$ \\
\hline 15 & p & KS & 180.7 & 28.23 & 47.08 & 6.59 & 1.0207 & 1.0627 & 1.0062 \\
\hline 31 & $\mathrm{Ga}$ & KS & 393.6 & 71.41 & 52.92 & 6.63 & 1.0580 & 1.0028 & 1.0581 \\
\hline & & & & 99.64 & 100.00 & & & & \\
\hline
\end{tabular}

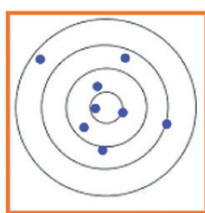

\section{(b)}

PeBaZAF 2 kcps

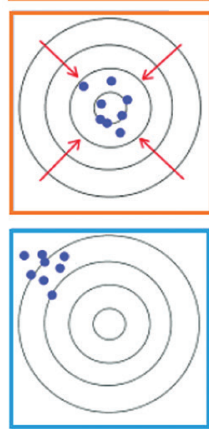

(c) PeBaZAF 200 kcps

(d) eZAF $200 \mathrm{kcps}$

a benefit to the PeBaZAF method. The test presented here shows that for 269 measurements of different multielement specimens, $95 \%$ of the results were within $\pm 13.7 \%$ of the known composition. This result is better than some published results for standardless EDS methods in $[9,17]$. Further development of this method is expected to improve the $\mathrm{P} / \mathrm{B}$ based standardless analysis to about $\pm 10 \%$ relative deviation

Figure 4: Effect of count rate. Internally calculated errors Err\% are marked with dark blue boxes (considering systematic+random errors). The top two analyses are for low-count measurements (30 seconds, $2 \mathrm{kcps}$ ): (a) eZAF method and (b) PeBaZAF method. Lower two analyses show results obtained with 100 times higher counts (200 kcps): (c) PeBaZAF and (d) eZAF. Both methods have similar total error for low counts. The PeBaZAF (P/B) method improves in precision and total error with higher counts. The eZAF method is hampered by the same high correction factors that limited accuracy at lower counts, and the total error improves only marginally.

the method is good (Figure 4a). The PeBaZAF method has better accuracy, but repeated values are more widely scattered, causing worse precision (Figure 4b). Under these conditions, the calculated total error (Err\%) is similar for both methods.

When the same sample is analyzed 100 times longer or with a count rate 100 times greater (for example, $200 \mathrm{kcps}$ with an $\mathrm{SDD}$ versus $2 \mathrm{kcps}$ with an $\mathrm{Si}(\mathrm{Li})$ detector), the precision improves for the PeBaZAF method (Figure 4c). This causes repeat analyses to be closer to the correct value. For the eZAF method the calculated error does not improve much, if at all, with higher count rates because the systematic errors in the correction factors still dominate (Figure $4 \mathrm{~d}$ ).

Figures 5 and 6 show the results of PeBaZAF standardless EDS analyses on 269 multi-element specimens containing various amounts of elements with atomic numbers from $\mathrm{Z}=6$ to $\mathrm{Z}=83$. For this group of test specimens, the relative deviation from the correct values was smaller than $\pm 13.7 \%$ for $95 \%$ of the results.

Thus, it is possible to improve the error factor for the PeBaZAF method with more counts in the spectrum, which leads to more counts in the background and better overall precision. Since the use of an SDD spectrometer makes spectrum acquisition at high count rates routine, the PeBaZAF method now has a significant advantage.

\section{Discussion}

The original PeBaZAF disadvantage, that it required higher than usual acquisition times, was reduced with the introduction of SDD detectors, where the count rate can be more than 2 orders of magnitude higher compared to the old $\mathrm{Si}(\mathrm{Li})$ detectors. This ability to offer more counts in the same collection time is clearly by employing a measured standards database (this standardless method uses "remote standards" [18]), which makes this method behave in a manner closer to a true standards-based quantitative analysis.

\section{Conclusion}

Two standardless methods were compared in light of the high count rate capabilities of the silicon drift detector (SDD). The disadvantage in precision typical of the PeBaZAF method is mitigated when using the high count rates available with the SDD. The test presented here shows that for 269 measurements

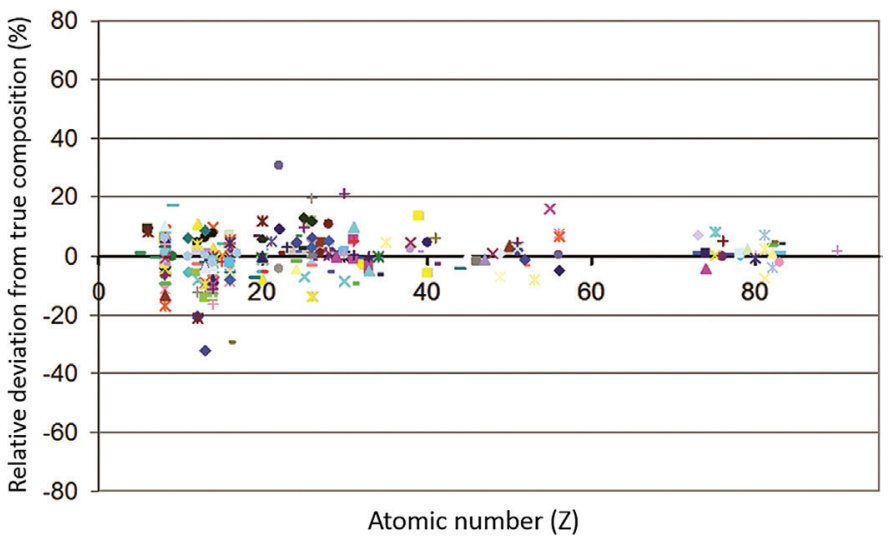

Figure 5: Results for 269 PeBaZAF standardless EDS analyses with an SDD on specimens containing various amounts of elements with atomic numbers from $Z=6$ to $Z=83$. The statistical evaluation of these results indicates that the relative deviation from correct values was better than $\pm 13.7 \%$ for $95 \%$ of results [14]. 


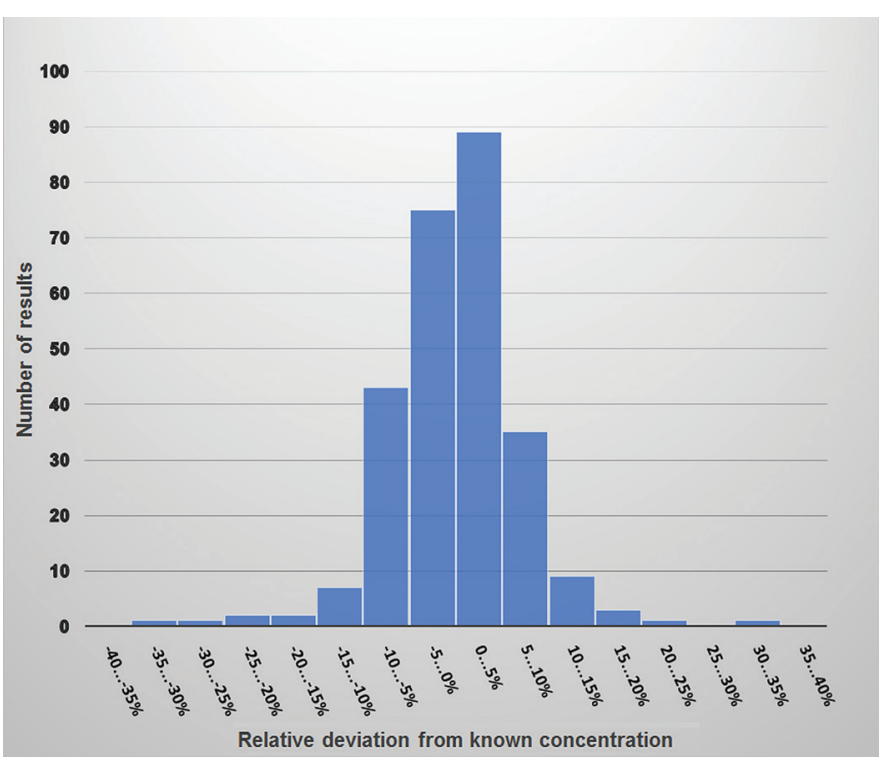

Figure 6: Error histogram for 269 PeBaZAF results showing the relative deviations from the true compositions. The central (largest) bar gives the number of results within the deviation range of from $0 \%$ to $5 \%$ of the true composition [14].

of different multi-element specimens, the PeBaZAF method yields $95 \%$ of the results within $\pm 13.7 \%$ of the known composition. This is better than certain other standardless methods. The eZAF method should gain improved accuracy from a stored database of standards measurements.

\section{MRS-6}

We are ISO-9000 certified and ISO-17025 accredited Microscopy Calibration Standard

Now you can calibrate better from $1,000 X$ to $1,000,000 X$ !

This is our fifth generation,

traceable,

magnification

reference standard

for all types (SEM,

FESEM, Optical,

STM, AFM, etc.) of microscopy. The

MRS-6 has multiple

$X$ and $Y$ pitch

patterns ranging

from $80 \mathrm{~nm}( \pm 3 \mathrm{~nm})$

to $2 \mu \mathrm{m}$ and $3 \mathrm{bar}$

targets from $80 \mathrm{~nm}$

to $3 \mu \mathrm{m}$. There is

also a STM test

pattern. Definition of

the $80 \mathrm{~nm}$ pitch

pattern is excellent.
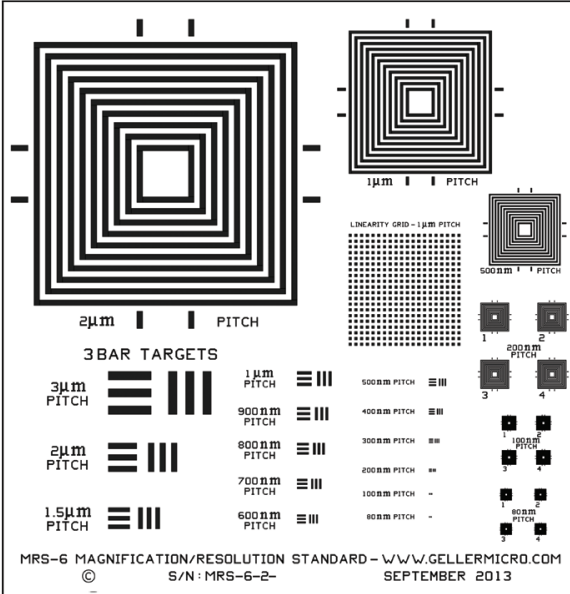

\section{GELLER}

MICROÅNALYTICAL LABORATORY, Inc.

426e BOSTON ST., TOPSFIELD, MA 01983-1216

TEL: 978 887-7000 FAX: 978-887-6671 www.GellerMicro.com

\section{Acknowledgements}

The author would like to acknowledge the contribution of all his EDAX colleagues, past and present, who have supported the development of experimental data and participated in the discussion of algorithms for this work.

\section{References}

[1] F Eggert, IOP Conf Ser.: Mater Sci Eng 7 (2010) 012007.

[2] F Eggert, Microchim Acta 155 (2006) 129-36.

[3] J Heckel and P Jugelt, X-Ray Spectrometry 13 (1984) $159-65$.

[4] F Eggert, Experim Techn Physik 33 (1985) 441-48.

[5] F Eggert et al., IOP Conf Ser: Mater Sci Eng 304 (2017) 012005.

[6] CG Ryan et al., Nucl Instrum Meth B 34(3) (1988) 396-402.

[7] F Eggert and W Scholz, Phys Stat Sol 97 (1986) K9-K13.

[8] WT Elam et al., Powder Diffr 25(2) (2010) F13.

[9] J Goldstein et al., Scanning Electron Microscopy and X-Ray Microanalysis, Third Edition, Springer, New York, 2003.

[10] G Love and VD Scott, J Phys D: Appl Phys 11 (1978) 1369-76.

[11] DA Sewell et al., J Phys D: Appl Phys 20 (1987) 1567-73.

[12] WT Elam et al., Radiat Phys Chem 63(2) (2002) 121-28.

[13] F Eggert and J Heckel, Experim Tech d Physik 34 (1986) 3, 201.

[14] F Eggert, Microsc Microanal 24 (Suppl 1) (2018) 201.

[15] M Wendt Krist Tech 13 (1978) 1259-75.

[16] F Eggert, Microsc Microanal 25 (Suppl 2) (2019) 560-61.

[17] J Goldstein et al., Scanning Electron Microscopy and X-Ray Microanalysis, Fourth Edition, Springer, New York, 2018.

[18] DE Newbury and NWM Ritchie, J Mater Sci 50 (2015) 493-518.

\section{SEM Scintillators \& Light Guides}

\section{Light Guide Recoating Services too!}

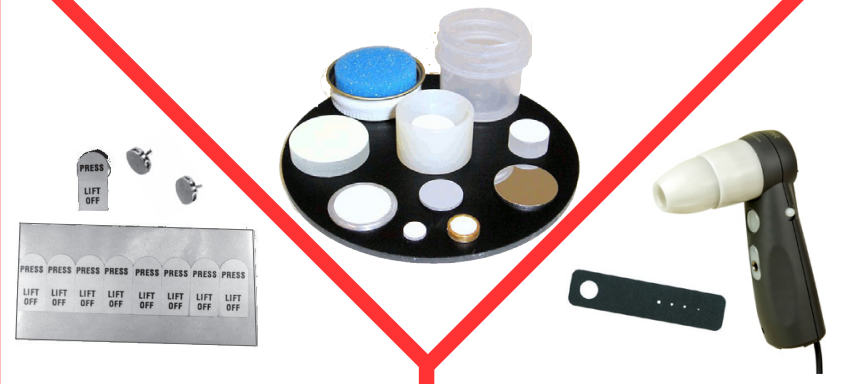

Conductive Adhesives, Apertures, ProScopes ${ }^{\mathrm{TM}}$ Coatings \& Tabs!

Vacuum Supplies

\& More!

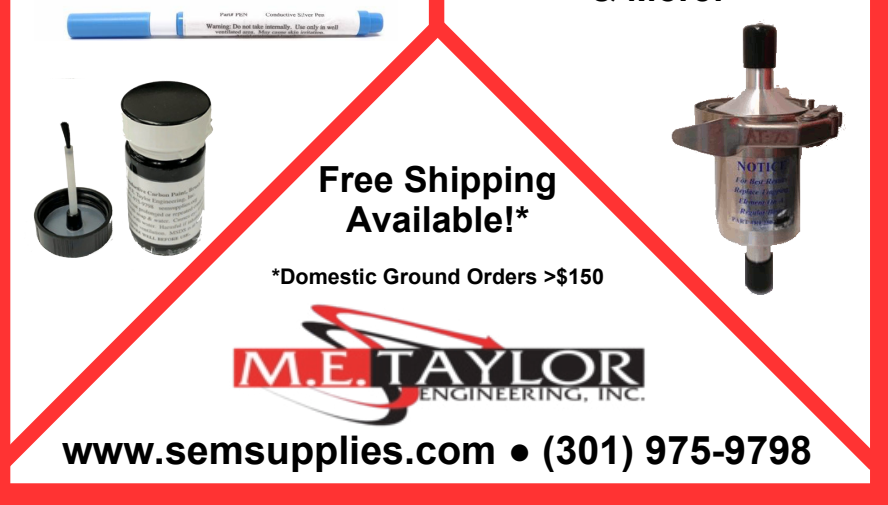

